\title{
Sessile serrated lesion detection rates during average risk screening colonoscopy: A systematic review and meta-analysis of the published literature
}

\section{(ㄷ)(우우}

Authors

Madhav Desai ${ }^{1}$, Joseph C. Anderson 2,3 , Michael Kaminski ${ }^{4,5,6}$, Viveksandeep Thoguluva Chandrasekar ${ }^{7}$, Jihan Fathallah $^{7}$, Cesare Hassan ${ }^{8}$, David Lieberman ${ }^{9}$, Prateek Sharma $^{1,7}$

Institutions

1 Department of Gastroenterology, Kansas City VA Medical Center, Kansas City, Missouri, United States

2 Department of Veterans Affairs Medical Center, White River Junction, Vermont, United States

3 The Geisel School of Medicine at Dartmouth, Hanover, New Hampshire, United States

4 Department of Gastroenterological Oncology, the Maria Sklodowska-Curie Memorial Cancer Center and Institute of Oncology, Warsaw, Poland

5 Department of Gastroenterology, Hepatology and Oncology, Medical Center for Postgraduate Education, Warsaw, Poland

6 Institute of Health and Society, University of Oslo, Oslo, Norway

7 Department of Gastroenterology and hepatology, University of Kansas Medical Center, Kansas City, Kansas, United States

8 Endoscopy Unit, Nuovo Regina Margherita Hospital, Rome, Italy

9 Department of Gastroenterology and Hepatology, Oregon Health and Science University, Portland, Oregon, United States

submitted 6.11 .2020

accepted after revision $\quad 10.12 .2020$

\section{Bibliography}

Endosc Int Open 2021; 09: E610-E620

DOI 10.1055/a-1352-4095

ISSN 2364-3722

(C) 2021. The Author(s).

This is an open access article published by Thieme under the terms of the Creative Commons Attribution-NonDerivative-NonCommercial License, permitting copying and reproduction so long as the original work is given appropriate credit. Contents may not be used for commercial purposes, or adapted, remixed, transformed or built upon. (https://creativecommons.org/licenses/by-nc-nd/4.0/)

Georg Thieme Verlag KG, Rüdigerstraße 14,

70469 Stuttgart, Germany

Corresponding author

Madhav Desai, MD, MPH, Department of Gastroenterology and hepatology, Kansas City VA Medical Center, 4801
Linwood Blvd, Kansas City, MO 64128, United States

Fax: +1-816-861-4700

drmadhavdesai@gmail.com

Supplementary material is available under https://doi.org/10.1055/a-1352-4095

\section{ABSTRACT}

Background and study aims Sessile serrated lesion (SSL) detection rate has been variably reported and unlike adenoma detection rate (ADR) is not currently a quality indicator for screening colonoscopy. Composite detection rates of SSL in patients undergoing average risk screening colonoscopy are not available.

Methods Electronic database search (Medline, Embase and Cochrane) was conducted for studies reporting detection rates of serrated polyps (SSL, Hyperplastic polyp, traditional serrated adenoma) among average risk subjects undergoing screening colonoscopy. Primary outcomes were pooled SDR (SSL detection rate) and proximal serrated polyp detection rate (PSPDR). Pooled proportion rates were calculated with $95 \% \mathrm{Cl}$ with assessment of heterogeneity $\left(\mathrm{I}^{2}\right)$. Publication bias, regression test and $95 \%$ prediction interval were calculated.

Results A total of 280,370 screening colonoscopies among average risk subjects that were eligible with $48.9 \%$ males and an average age of 58.7 years $( \pm 3.2)$. The pooled SDR was available from 16 studies: $2.5 \%(1.8 \%-3.4 \%)$ with significant heterogeneity $\left(I^{2}=98.66 \%\right)$ and the $95 \%$ prediction interval ranging from $0.6 \%$ to $9.89 \%$. When analysis was restricted to large $(n>1000)$ and prospective studies $(n=4)$, SDR was $2 \%(1.1 \%-3.3 \%)$. Pooled PSPDR was $10 \%(8.5 \%-$ $11.8 \% ; 12$ studies). There was evidence of publication bias $(P<0.01)$.

Conclusion Definitions of SSL have been varying over years and there exists significant heterogeneity in prevalence reporting of serrated polyps during screening colonoscopy. Prevalence rate of $2 \%$ for SSL and $10 \%$ for proximal serrated polyps could serve as targets while robust high-quality data is awaited to find a future benchmark showing reduction in colorectal cancer arising from serrated pathway. 


\section{Introduction}

Colorectal cancer (CRC) is the third most common cancer worldwide and screening colonoscopy is the current strongest weapon in the battle to prevent it $[1,2]$. Adenomas were long thought to be the lone precursor lesion for development of CRC and therefore, adenoma detection rate (ADR) was established as a quality criterion for screening colonoscopy. Recent evidence has shown that ADR is inversely associated with the risk of post colonoscopy CRC incidence and mortality [3]. High detectors can reduce such risk by $50 \%$ to $90 \%$ [4]. Therefore, ADR has become a crucial benchmark for endoscopist performance and the target for quality improvement [3]. Serrated colon lesions, previously thought to be benign polyps, have emerged as another key pathway contributing to CRC development [57]. Thus, with improvement in endoscopist achievement of benchmark ADRs and serrated lesions becoming widely recognized, the focus is now on serrated polyp detection with the goal of improving overall quality of colonoscopy to reduce serrated lesion-related post-colonoscopy CRC.

Emerging data are suggestive of suboptimal efficacy of colonoscopy in reducing the rates of proximal colon cancer (proximal to splenic flexure) which might be partly due to missed serrated lesions, which tend to be in the proximal colon [8]. In addition, post-colonoscopy cancers are more likely to have characteristics of serrated pathway including microsatellite instability, characteristic histological pattern and proximal location [9, 10]. Serrated lesions represent a heterogeneous group of colorectal lesions that includes hyperplastic polyps (HP), sessile serrated lesion (SSL; previously SSA/P), and traditional serrated adenoma (TSA) [5, 11]. HP are still believed to be benign lesions with no risk of malignant transformation while SSLs and TSAs have been considered precursor lesions. SSLs progress via the serrated pathway to CRC [12]. CRCs derived from serrated pathways constitute $20 \%$ to $30 \%$ of all CRCs $[5,12]$. SSLs are flat or sessile, usually covered by a mucous cap and generally located in the proximal colon. Because of their subtle morphology, they are difficult to detect and even when detected, are often incompletely resected. In addition, some SSL are reported to progress to invasive cancer in a short period of time [11]. Therefore, detection of SSLs is crucial to reduce interval CRC and CRC-related death, and efforts to increase the SSL detection rate (SDR), like ADR, could potentially decrease CRC mortality. Because there is significant variation in pathologist interpretation of serrated polyps in the proximal colon, the proximal serrated polyp detection rate (PSPDR) is also an important parameter that has shown to correlate very well with SDR.

Studies to date variably report detection rate of SSL and proximal serrated lesions during average risk screening colonoscopy. Composite SDR and PSPDR for screening colonoscopy are not known to guide endoscopists. Such summary estimates would help track performance measures and for future search to understand its impact on interval colon cancer. Our aim was to conduct a systematic review and meta-analysis of available literature to derive the pooled detection rates for SDR and PSPDR in patients undergoing average risk screening colonoscopy.

\section{Methods}

This systematic review and pooled-analysis was performed per the PRISMA and MOOSE guidelines [13]. Study flow diagram depicting literature search, application of inclusion and exclusion of studies, screening of articles, review and final selection is shown in $>$ Fig. 1.

\section{Data Sources and searches}

A comprehensive literature search for studies of screening colonoscopies reporting serrated polyp (as defined per WHO consensus definition) information was performed in MEDLINE, EMBASE and the Cochrane Central Register of Controlled Trials (from inception to July 15, 2020). Search terms for serrated polyps were used to find articles of relevance without any language restriction: (((Serrated polyp [MeSH Terms]) OR (Serrated lesion [MeSH Terms])) OR (Sessile serrated polyp [MeSH Terms])) OR (sessile serrated adenoma [MeSH Terms]). Initial literature search was conducted by two authors (M.D. and J.F.) and results were compared, and data was merged for study extraction.

\section{Study selection}

Two reviewers (M.D. and J.F.) independently screened the search results in all databases by title and abstract to screen eligible studies after removing duplicates. Then all the eligible full-text articles were reviewed in depth for final eligibility and excluded when any criteria for exclusion were noted. Any prospective or retrospective studies reporting information on findings of screening colonoscopy in average risk individuals and number of patients with at least one serrated polyp and SSL were included. Studies that were in abstract form only (in lack for variables of interest for in depth analysis), case reports and case series, review articles, editorials and studies of diagnostic and surveillance colonoscopy were excluded. Studies where fecal immunochemical test (FIT) and other screening modalities (i. e. flexible sigmoidoscopy, computed tomography colonography) were used followed by colonoscopies (for investigation of positive results) were excluded as well. Studies with subjects undergoing screening colonoscopy in one arm and outcomes separately reported were eligible. When there was discrepancy regarding the study eligibility, consensus was achieved by article review by senior author (P.S.).

\section{Definitions}

The SDR was defined as the number of patients who had at least one SSL found on screening colonoscopy divided by total number of patients, respectively. The PSPDR was defined as the number of patients with at least one proximal serrated polyp (SSL, HP and/or TSA). Clinically significant (or relevant) serrated polyps included any SSL, TSA or large HP $(>1 \mathrm{~cm})$ found anywhere in the colon or hyperplastic polyp $>5 \mathrm{~mm}$ in the proximal colon only divided by the total number of screening colonoscopies, respectively. 


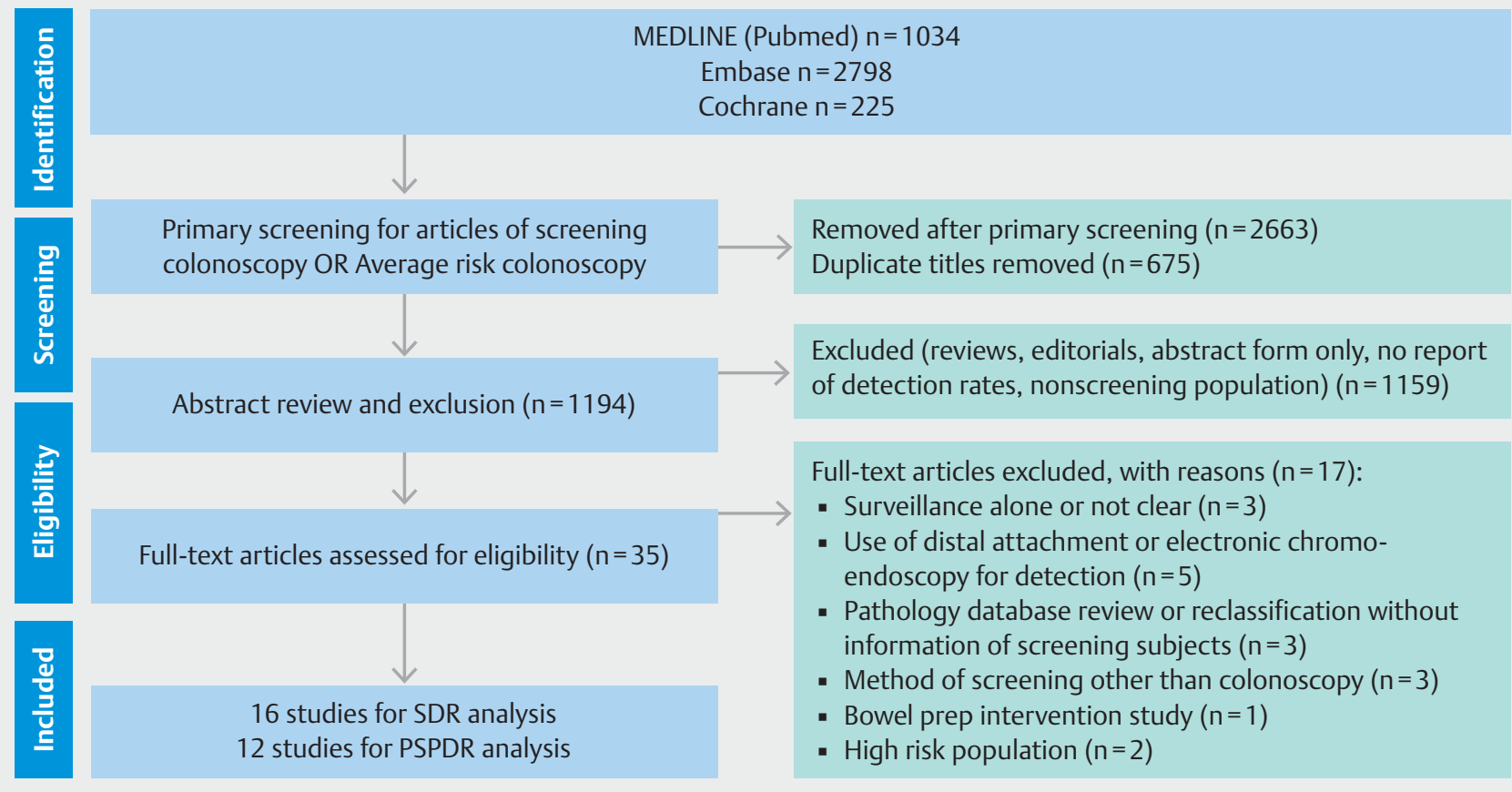

Fig. 1 Study flow diagram.

\section{Data extraction and Quality assessment}

Two reviewers (M.D. and J.F.) independently assessed each study for eligibility and extracted data on characteristics of the study including article name, study type, year, number of centers, type of colonoscopy, number of patients undergoing screening colonoscopy, gender, age, ethnicity, smoking status, body mass index (BMI), bowel prep (type and result) and reported outcomes including number of patients with at least one SSL, proximal SSL, any serrated polyp, any proximal serrated polyp, large serrated polyp and large SSL $(>1 \mathrm{~cm})$, SSL with dysplasia, TSA, and advanced adenoma. Per patient data were collected as reported from the studies. Extracted data were arranged into tables for verification and accuracy prior to analysis.

\section{Risk of bias assessment}

Information on the methodological quality of each study was recorded and quality assessment was performed using the Newcastle-Ottawa Scale (NOS) [14] for cohort studies. Cochrane risk of bias tool [15] was used for quality assessment of randomized controlled trials (RCTs).

\section{Statistical analysis}

As the primary objective was to examine prevalence of detection of any SSL and any proximal serrated lesion in a screening colonoscopy exam, primary outcome of this analysis was pooled rate of SDR and PSPDR from available studies. Individual screening colonoscopy studies might have reported detection of these outcomes as primary or secondary outcomes. For purpose of analysis, SDR was calculated as number of colonoscopy with at least one SSL divided by number of all average risk screening colonoscopies. PSPDR, similarly, was counted as number of colonoscopy with at least one proximal serrated polyp divided by number of all average risk screening colonoscopies.

Sensitivity analysis was performed for: SDR from prospective studies alone, SDR of studies with more than 1000 patients alone and those with prospective studies with more than 1000 patients. Similar analyses were performed for PSPDR. Studies with greater than 1000 patients may represent more precise population distribution and therefore were analyzed separately in order to minimize any heterogeneity. Analysis was restricted to prospective studies alone to understand any bias from retrospective chart-based data that may interfere with pooled estimate. Prediction interval with $95 \%$ confidence interval $(\mathrm{Cl})$ was also derived for pooled estimate of SDR from all studies to assess true population variation.

Additional outcomes that were analyzed were: pooled detection rate of any serrated polyp (SSL, HP and/or TSA), large serrated polyp ( $\geq 1 \mathrm{~cm}$, proximal SSL, large SSL $(\geq 1 \mathrm{~cm})$ and SSL with dysplasia. Pooled TSA detection rate and synchronous advanced adenoma detection rate were calculated as well. Pooled detection rate of clinically significant serrated polyp was calculated as reported from studies directly. We also reviewed literature for incidence of new diagnosis of serrated polyposis syndrome after screening colonoscopy.

Study and patient characteristics were recorded in the form of tables and data were presented as frequencies (\%) or average (mean or median as available from the study) rates with range. Summary estimates are calculated from effect sizes from individual studies using a random-effects model a priori per Co- 
chrane handbook of systematic reviews assuming detection of any serrated lesion during a screening colonoscopy being a common effect across all studies. Summary estimates were represented as pooled proportions with $95 \%$ confidence intervals $(\mathrm{Cl})$. Heterogeneity $\left(\mathrm{I}^{2}\right)$ among the studies was calculated where $\mathrm{I}^{2}$ values of $25 \%, 50 \%$, and $75 \%$ were considered as low, moderate, and high heterogeneity, respectively. Risk of any publication bias was examined with funnel plot. Egger's regression was used to test for funnel plot asymmetry. The 95\%Prediction interval was also calculated to assess real world distribution of the pooled SDR found from this analysis. $P<0.05$ was considered statistically significant. Comprehensive meta-analysis v3 (Biostat, Englewood, New Jersey, United States) was used for this pooled analysis.

\section{Results}

A total of 4057 records were found from the initial search of the electronic databases of which after removal of duplicates and application of primary screening criteria, there were 1194 records for review ( $\triangleright$ Fig. 1 ). From this, after review of abstract level data, there were 35 articles that were reviewed. We found 16 eligible studies [16-30] reporting SDR for pooled analysis and 12 studies $[17,19-22,25,29,31-35]$ for PSPDR analysis (giving total 22 studies). Studies from the same investigators were included only when one primary outcome was reported in one publication and another in a different one [16,35].

There were a total of 280,370 eligible screening colonoscopies providing information on serrated lesions: $48.9 \%$ males; average age 58.7 years $( \pm 3.2)$. Information on smoking use was available only in three studies ( $11 \%$ of subjects among 14752 undergoing screening) $[16,18,34]$. Information on BMI was reported in only four studies $[16,18,26,34]$. Bowel prep was reported in 15 studies with nine studies that excluded patients with poor and inadequate bowel prep. There were total of 12 studies that were conducted in the United States while the rest were either from Europe $(n=7)$, Asia (China:1; Taiwan:1) or Argentina $(n=1)$. This and patient characteristics are listed in $>$ Table 1 and $>$ Table 2 .

\section{Study quality}

There were eight prospective studies of which two were RCTs $[19,21]$ and six were prospective cohort studies $[17,18,22$, $23,31,36]$. The remaining nine studies were retrospective cohort studies. The results of study quality assessment are shown in Supplementary Table 1.

\section{Pooled SDR}

In a total of 16 studies of 234,459 subjects undergoing screening colonoscopy, at least one SSL was found in 12,677 subjects. The pooled estimate of SDR was $2.5 \%$ (95\% Cl: $1.8 \%-3.4 \%$ ) with a high level of heterogeneity among included studies ( $\mathrm{I}^{2}$ of $98.66 \%$ ) ( Fig. 2). The 95\%Prediction interval for SDR was $0.6 \%$ to $9.89 \%$.

When analysis was restricted to studies with more than 1000 patients $(n=13)$, the pooled SDR was $2.4 \%(95 \% \mathrm{Cl} 1.7 \%-3.4 \%$; Supplementary Fig. 1). When analysis was restricted to pro- spective studies alone $(n=7)$, the pooled SDR was $2.3 \%(95 \%$ $\mathrm{Cl}$ : 1.5\%-3.6\%) (Supplementary Fig.2). Finally, when information for prospective studies with more than 1000 patients only was included $(n=4)$, the pooled SDR was $2 \%(95 \% 1.1 \%-3.3 \%$; $P<0.01)$ ( $>$ Table 3$)$.

\section{Pooled PSPDR}

In 12 studies reporting PSPDR, there were 76,950 subjects undergoing screening colonoscopy and at least 1 proximal serrated polyp was found in 8,198 subjects. The pooled estimate of PSPDR was $10 \%(95 \% \mathrm{Cl} 8.5 \%-11.8 \%)$ with high heterogeneity $(\mathrm{I} 2=98.17 \%)$ ( $\mathrm{Fig} .3)$. When studies were restricted to those with more than 1000 subjects $(n=11)$, the pooled PSPDR estimate was $10 \%$ (8.4\%-11.9\%) (Supplementary Fig.3). When data was further restricted to prospective studies with more than 1000 subjects $(n=3)$, pooled PSPDR was $11.2 \%(95 \% \mathrm{Cl}$ $9.3 \%-13.5 \%$; $P<0.01$ ) ( Table 3 ).

\section{Secondary outcomes}

Pooled detection rate of other outcomes are presented in $>\mathbf{T a}$ ble 3 and corresponding forest plots are demonstrated in Supplementary Fig.4-11.

TSA prevalence during screening colonoscopy was a rare event with rate of $0.2 \%$ only $(0.1 \%-0.6 \%)$. Prevalence of SSL with dysplasia with low with rate of $0.8 \%(0.4 \%-1.6 \%)$ while synchronous advanced ADR among available studies was $19.1 \%$ (15.6\%-23.1\%). Only one prospective study reported incidence of serrated polyposis syndrome found after index colonoscopy at $0.09 \%$ (12 cases of 13,787 screened) [22]. $P<0.01$ for all pooled estimates.

\section{Publication bias}

The funnel plot diagram is shown in > Fig. 4. Asymmetry was detected in the funnel plot for studies of SDR. Egger's regression test showed test of significance confirming there was evidence of publication bias among available studies (intercept $7.3 ; P<0.01)$.

\section{Discussion}

SSL and proximal serrated lesion are clinically important lesions found during average risk screening colonoscopy and precise prevalence rates are not known. Our analysis found that of 100 subjects underlying screening colonoscopy, at least four could be found to have an SSL lesion while 10 of them would harbor a proximally located serrated polyp. On the other hand, prevalence of TSA and SSL with dysplasia is quite low. We also found that the pooled rate of SSL would vary extremely in the real world ranging from $<1 \%$ to around $10 \%$ which seems reflective of high heterogeneity we noted in the available data. While there remains concern regarding varying definitions over last several years and heterogeneity in reporting them, this is the first reporting of pooled estimates from the available data. Majority of the population based large cohort studies were conducted before the World Health Organization (WHO) classification of serrated lesions and hence their exact distribution and importance was not well known until recently. The United 
- Table1 Study characteristics and outcomes.

\begin{tabular}{|c|c|c|c|c|c|c|c|c|}
\hline Study & $\begin{array}{l}\text { Type of } \\
\text { study }\end{array}$ & Country & $\begin{array}{l}\text { Screen- } \\
\text { ing co- } \\
\text { lonos- } \\
\text { copies }\end{array}$ & $\begin{array}{l}\text { Serrated } \\
\text { polyp detec- } \\
\text { tion rate }\end{array}$ & PSPDR & SDR & $\begin{array}{l}\text { Hyperplastic } \\
\text { polyp detec- } \\
\text { tion rate }\end{array}$ & $\begin{array}{l}\text { Traditional } \\
\text { serrated } \\
\text { adenoma } \\
\text { detection } \\
\text { rate }\end{array}$ \\
\hline $\begin{array}{l}\text { Chang } 2016 \\
\text { [18] }\end{array}$ & Prospective & Taiwan & 6198 & NA & NA & $89(1.4 \%)$ & $11(1.9 \%)$ & NA \\
\hline $\begin{array}{l}\text { Ross } 2015 \\
{[26]}\end{array}$ & $\begin{array}{l}\text { Retrospec- } \\
\text { tive }\end{array}$ & USA & 2833 & NA & NA & $232(8.1 \%)$ & NA & NA \\
\hline $\begin{array}{l}\text { Sanaka } 2014 \\
\text { [27] }\end{array}$ & $\begin{array}{l}\text { Retrospec- } \\
\text { tive }\end{array}$ & USA & 2167 & NA & NA & $39(1.8 \%)$ & NA & NA \\
\hline $\begin{array}{l}\text { Pereyra } 2014 \\
\text { [36] }\end{array}$ & Prospective & Argentina & 272 & NA & NA & $21(7.7 \%)$ & NA & NA \\
\hline $\begin{array}{l}\text { Hazewinkel } \\
2014 \text { [19] }\end{array}$ & $\mathrm{RCT}$ & $\begin{array}{l}\text { Nether- } \\
\text { lands }\end{array}$ & 1426 & $388(27.2 \%)$ & $174(12.2 \%)$ & $68(4.8 \%)$ & $33(23.8 \%)$ & $1(0.1 \%)$ \\
\hline $\begin{array}{l}\text { Leung } 2012 \\
\text { [33] }\end{array}$ & $\begin{array}{l}\text { Retrospec- } \\
\text { tive }\end{array}$ & China & 1282 & $274(21.4 \%)$ & $92(7.1 \%)$ & NA & NA & NA \\
\hline $\begin{array}{l}\text { Kahi } 2011 \\
{[32]}\end{array}$ & $\begin{array}{l}\text { Retrospec- } \\
\text { tive }\end{array}$ & USA & 6681 & 3984 (59.6\%) & $1238(18.5 \%)$ & NA & NA & NA \\
\hline $\begin{array}{l}\text { Hetzel } 2010 \\
{[20]}\end{array}$ & $\begin{array}{l}\text { Retrospec- } \\
\text { tive }\end{array}$ & USA & 7192 & NA & 684 & $46(0.6 \%)$ & $842(11.7 \%)$ & NA \\
\hline $\begin{array}{l}\text { Buda } 2012 \\
\text { [17] }\end{array}$ & Prospective & Italy & 985 & NA & 103 & $23(2.3 \%)$ & $45(4.6 \%)$ & $6(0.6 \%)$ \\
\hline $\begin{array}{l}\text { Ladabaum } \\
2016 \text { [23] }\end{array}$ & Prospective & USA & 509 & $13(2.55 \%)$ & NA & 6 & NA & NA \\
\hline $\begin{array}{l}\text { ljspeert } 2016 \\
{[22]}\end{array}$ & Prospective & Europe & 13787 & $3676(26.6 \%)$ & $1338(9.7 \%)$ & $341(2.4 \%)$ & NA & $100(0.7 \%)$ \\
\hline $\begin{array}{l}\text { ljspeert } 2016 \\
{[21]}\end{array}$ & RCT & $\begin{array}{l}\text { Nether- } \\
\text { lands }\end{array}$ & 1276 & $399(31.3 \%)$ & NA & $8(6.5 \%)$ & $316(24.7 \%)$ & $1(0.08 \%)$ \\
\hline $\begin{array}{l}\text { Schachschal } \\
2016[28]\end{array}$ & $\begin{array}{l}\text { Retrospec- } \\
\text { tive }\end{array}$ & Germany & 1069 & $170(15.9 \%)$ & NA & $7(0.6 \%)$ & $165(15.4 \%)$ & 0 \\
\hline $\begin{array}{l}\text { Schreiner } \\
2010[34]\end{array}$ & $\begin{array}{l}\text { Retrospec- } \\
\text { tive }\end{array}$ & USA & 3121 & $801(25.6 \%)$ & $348(11.1 \%)$ & NA & NA & NA \\
\hline $\begin{array}{l}\text { Anderson } \\
2018[16]\end{array}$ & $\begin{array}{l}\text { Retrospec- } \\
\text { tive }\end{array}$ & USA & 5433 & $1016(18.7 \%)$ & NA & $181(3.3 \%)$ & $1262(23.2 \%)$ & NA \\
\hline $\begin{array}{l}\text { Anderson } \\
2017[35]\end{array}$ & $\begin{array}{l}\text { Retrospec- } \\
\text { tive }\end{array}$ & USA & 29,960 & NA & $3236(10.8 \%)$ & NA & NA & NA \\
\hline $\begin{array}{l}\text { Parikh } 2017 \\
\text { [25] }\end{array}$ & $\begin{array}{l}\text { Retrospec- } \\
\text { tive }\end{array}$ & USA & 4151 & NA & $254(6.1 \%)$ & $179(4.3 \%)$ & NA & NA \\
\hline $\begin{array}{l}\text { Shaukat } \\
2019[30]\end{array}$ & $\begin{array}{l}\text { Retrospec- } \\
\text { tive }\end{array}$ & USA & 180150 & NA & NA & 11170 (6.2\%) & NA & NA \\
\hline $\begin{array}{l}\text { Schramm } \\
2018 \text { [29] }\end{array}$ & $\begin{array}{l}\text { Retrospec- } \\
\text { tive }\end{array}$ & Germany & 4161 & $807(19.4 \%)$ & $308(7.4 \%)$ & $124(3 \%)$ & $720(17.1 \%)$ & $4(0.1 \%)$ \\
\hline $\begin{array}{l}\text { de Wijker- } \\
\text { slooth } 2013 \\
{[31]}\end{array}$ & Prospective & $\begin{array}{l}\text { Nether- } \\
\text { lands }\end{array}$ & 1354 & NA & $167(12 \%)$ & NA & NA & NA \\
\hline $\begin{array}{l}\text { Klair } 2020 \\
{[47]}\end{array}$ & $\begin{array}{l}\text { Retrospec- } \\
\text { tive }\end{array}$ & USA & 3513 & NA & NA & $\begin{array}{l}\text { CsSPDR: } 362 \\
(10.3 \%)\end{array}$ & NA & NA \\
\hline $\begin{array}{l}\text { Mandaliya } \\
2019 \text { [24] }\end{array}$ & $\begin{array}{l}\text { Retrospec- } \\
\text { tive }\end{array}$ & USA & 2850 & NA & $256(9 \%)$ & $68(2.4 \%)$ & NA & NA \\
\hline
\end{tabular}


- Table 2 Patient characteristics

\begin{tabular}{|c|c|c|c|c|c|c|c|c|c|}
\hline Study & $\begin{array}{l}\text { Total } \\
\text { Sub- } \\
\text { jects }\end{array}$ & $\begin{array}{l}\text { Males/ } \\
\text { Total } \\
(\%)\end{array}$ & $\begin{array}{l}\text { Ethnici- } \\
\text { ty }\end{array}$ & $\begin{array}{l}\text { Age } \\
\text { (mean or } \\
\text { median } \\
\text { with SD } \\
\text { or IQR) }\end{array}$ & Bowel prep & $\begin{array}{l}\text { Family his- } \\
\text { tory of co- } \\
\text { lon cancer } \\
\text { in } 1^{\text {st }} \text { or } 2^{\text {nd- }} \\
\text { degree rela- } \\
\text { tive }\end{array}$ & $\begin{array}{l}\text { Inspec- } \\
\text { tion } \\
\text { time }\end{array}$ & $\begin{array}{l}\text { Cecal } \\
\text { intuba- } \\
\text { tion } \\
\text { rate }\end{array}$ & $\begin{array}{l}\text { Withdrawal } \\
\text { time (Mean } \\
\text { or median } \\
\text { with } \pm S D \text { or } \\
\text { IQR) }\end{array}$ \\
\hline $\begin{array}{l}\text { Chang } 2016 \\
\text { [18] }\end{array}$ & 6198 & $\begin{array}{l}3165 \\
(51.1 \%)\end{array}$ & NA & 59 & $\begin{array}{l}\text { Poor Prep } \\
(n=552 ; 9 \%)\end{array}$ & NA & NA & $99.30 \%$ & NA \\
\hline $\begin{array}{l}\text { Ross } 2015 \\
{[26]}\end{array}$ & 2833 & $\begin{array}{l}1003 \\
(35.4 \%)\end{array}$ & $\begin{array}{l}\text { Cauca- } \\
\text { sians (n } \\
=1926 ; \\
68 \%)\end{array}$ & 55 & NA & $283(10 \%)$ & NA & NA & NA \\
\hline $\begin{array}{l}\text { Sanaka } \\
2014 \text { [27] }\end{array}$ & 2167 & $\begin{array}{l}1129 \\
(52 \%)\end{array}$ & NA & 59 & $\begin{array}{l}\text { Excellent } \\
\text { prep } \\
(\mathrm{N}=1029) ; \\
\text { Good prep } \\
(\mathrm{n}=1138) \\
{[100 \%]}\end{array}$ & NA & NA & NA & NA \\
\hline $\begin{array}{l}\text { Pereyra } 2014 \\
\text { [36] }\end{array}$ & 272 & $\begin{array}{l}148 \\
(54 \%)\end{array}$ & NA & 62 & $\begin{array}{l}\text { Inadequate } \\
\text { prep were } \\
\text { excluded }\end{array}$ & NA & NA & NA & NA \\
\hline $\begin{array}{l}\text { Hazewinkel } \\
2014 \text { [19] }\end{array}$ & 1426 & $\begin{array}{l}727 \\
(51 \%)\end{array}$ & NA & 60 & $\begin{array}{l}\text { Median Ot- } \\
\text { tawa bowel } \\
\text { prep score } 5 \\
\text { (IQR 3-8) }\end{array}$ & NA & NA & $98.70 \%$ & $\begin{array}{l}10(8-15) \\
\min \end{array}$ \\
\hline $\begin{array}{l}\text { Leung } 2012 \\
\text { [33] }\end{array}$ & 1282 & $\begin{array}{l}620 \\
(48.4 \%)\end{array}$ & $\begin{array}{l}\text { Asian } \\
\text { (China) } \\
100 \%\end{array}$ & 49.1 & NA & Excluded & NA & $99.30 \%$ & NA \\
\hline $\begin{array}{l}\text { Kahi } 2011 \\
\text { [32] }\end{array}$ & 6681 & $\begin{array}{l}3273 \\
(49 \%)\end{array}$ & NA & 58.9 & NA & Excluded & NA & NA & NA \\
\hline $\begin{array}{l}\text { Hetzel } 2010 \\
{[20]}\end{array}$ & 7192 & $\begin{array}{l}3165 \\
(44 \%)\end{array}$ & NA & 57 & NA & NA & NA & NA & NA \\
\hline $\begin{array}{l}\text { Buda } 2012 \\
\text { [17] }\end{array}$ & 985 & $\begin{array}{l}375 \\
(38 \%)\end{array}$ & NA & 53 & $\begin{array}{l}\text { Poor prep } \\
\text { were exclud- } \\
\text { ed }\end{array}$ & Excluded & $\begin{array}{l}\text { Total } \\
\text { proce- } \\
\text { dure } \\
\text { time } \\
23.1 \\
( \pm 6.4) \\
\text { min }\end{array}$ & NA & $\begin{array}{l}6.9( \pm 1.3) \\
\min \end{array}$ \\
\hline $\begin{array}{l}\text { Ladabaum } \\
2016 \text { [23] }\end{array}$ & 509 & $\begin{array}{l}253 \\
(49.7 \%)\end{array}$ & $\begin{array}{l}\text { Cauca- } \\
\text { sians (n } \\
=311 ; \\
61 \%)\end{array}$ & $58(52-65)$ & $\begin{array}{l}\text { Only good } \\
\text { prep includ- } \\
\text { ed }\end{array}$ & NA & NA & NA & NA \\
\hline $\begin{array}{l}\text { ljspeert } \\
2016 \text { [22] }\end{array}$ & 13787 & $\begin{array}{l}6568 \\
(47.6 \%)\end{array}$ & NA & $50-75$ & $\begin{array}{l}\text { Adequate } \\
\text { bowel prep } \\
(n=12693 ; \\
92 \%)\end{array}$ & NA & NA & $\begin{array}{l}13480 \\
(98 \%)\end{array}$ & NA \\
\hline $\begin{array}{l}\text { ljspeert } \\
2016 \text { [21] }\end{array}$ & 1276 & $\begin{array}{l}652 \\
(51 \%)\end{array}$ & NA & $\begin{array}{l}60.1 \\
( \pm 6.2)\end{array}$ & NA & NA & NA & NA & NA \\
\hline $\begin{array}{l}\text { Schachschal } \\
2016[28]\end{array}$ & 1069 & NA & NA & NA & NA & NA & NA & NA & NA \\
\hline $\begin{array}{l}\text { Schreiner } \\
2010 \text { [34] }\end{array}$ & 3121 & $\begin{array}{l}3021 \\
(96.8 \%)\end{array}$ & $\begin{array}{l}\text { Cauca- } \\
\text { sians: n } \\
=2607 ; \\
83 \%)\end{array}$ & NA & NA & NA & NA & NA & NA \\
\hline
\end{tabular}




\begin{tabular}{|c|c|c|c|c|c|c|c|c|c|}
\hline Study & $\begin{array}{l}\text { Total } \\
\text { Sub- } \\
\text { jects }\end{array}$ & $\begin{array}{l}\text { Males| } \\
\text { Total } \\
(\%)\end{array}$ & $\begin{array}{l}\text { Ethnici- } \\
\text { ty }\end{array}$ & $\begin{array}{l}\text { Age } \\
\text { (mean or } \\
\text { median } \\
\text { with SD } \\
\text { or IQR) }\end{array}$ & Bowel prep & $\begin{array}{l}\text { Family his- } \\
\text { tory of co- } \\
\text { lon cancer } \\
\text { in } 1^{\text {st }} \text { or } 2^{\text {nd }} \\
\text { degree rela- } \\
\text { tive }\end{array}$ & $\begin{array}{l}\text { Inspec- } \\
\text { tion } \\
\text { time }\end{array}$ & $\begin{array}{l}\text { Cecal } \\
\text { intuba- } \\
\text { tion } \\
\text { rate }\end{array}$ & $\begin{array}{l}\text { Withdrawal } \\
\text { time (Mean } \\
\text { or median } \\
\text { with } \pm S D \text { or } \\
\text { IQR) }\end{array}$ \\
\hline $\begin{array}{l}\text { Anderson } \\
2018 \text { [16] }\end{array}$ & 5433 & $\begin{array}{l}2702 \\
(49.7 \%)\end{array}$ & NA & $61(54-74)$ & $\begin{array}{l}\text { Poor prep } \\
(n=237) \text { ex- } \\
\text { cluded }\end{array}$ & $988(18 \%)$ & NA & NA & NA \\
\hline $\begin{array}{l}\text { Anderson } \\
2017 \text { [35] }\end{array}$ & 29960 & $\begin{array}{l}14231 \\
(47.5 \%)\end{array}$ & NA & $59(53-66)$ & $\begin{array}{l}\text { Poor prep } \\
\text { excluded }\end{array}$ & Excluded & NA & NA & $\begin{array}{l}<9 \min (57 \% \\
\text { endos- } \\
\text { copists) }\end{array}$ \\
\hline $\begin{array}{l}\text { Parikh } 2017 \\
\text { [25] }\end{array}$ & 4151 & $\begin{array}{l}1944 \\
(46.8 \%)\end{array}$ & $\begin{array}{l}\text { Cauca- } \\
\text { sians: } \mathrm{n} \\
=3334 \\
80 \%\end{array}$ & 60 & $\begin{array}{l}\text { Excellent } \\
\text { prep }(n= \\
652) ; \text { Good } \\
\text { prep }(n= \\
2675), \text { Ade- } \\
\text { quate }(n= \\
824)[100 \%]\end{array}$ & NA & NA & NA & NA \\
\hline $\begin{array}{l}\text { Shaukat } \\
2019 \text { [30] }\end{array}$ & 180150 & NA & NA & $60.1( \pm 9)$ & $\begin{array}{l}\text { Only ade- } \\
\text { quate preps } \\
\text { included }\end{array}$ & NA & NA & NA & NA \\
\hline $\begin{array}{l}\text { Schramm } \\
2018 \text { [29] }\end{array}$ & 4161 & $\begin{array}{l}2022 \\
(48.6 \%)\end{array}$ & NA & $62(56-69)$ & $\begin{array}{l}\text { Adequate } \\
\text { prep }(n= \\
4024 ; 96.7 \\
\%)\end{array}$ & NA & NA & NA & NA \\
\hline $\begin{array}{l}\text { Wijkerslooth } \\
2013 \text { [31] }\end{array}$ & 1354 & $\begin{array}{l}689 \\
(51 \%)\end{array}$ & NA & $60(55-65)$ & $\begin{array}{l}\text { Ottawa bow- } \\
\text { el prep score } \\
5(3-8)\end{array}$ & NA & NA & NA & $\begin{array}{l}10(8-15) \\
\text { minutes }\end{array}$ \\
\hline $\begin{array}{l}\text { Klair } 2020 \\
{[47]}\end{array}$ & 3513 & $\begin{array}{l}1585 \\
(45 \%)\end{array}$ & $\begin{array}{l}\text { Cauca- } \\
\text { sians } \\
\mathrm{n}=2740 \\
(78 \%)\end{array}$ & $\begin{array}{l}56.8( \\
+/-7.4)\end{array}$ & $\begin{array}{l}\text { Only ade- } \\
\text { quate bowel } \\
\text { prep includ- } \\
\text { ed }\end{array}$ & Excluded & NA & NA & $\begin{array}{l}10(9-12) \\
\text { minutes }\end{array}$ \\
\hline $\begin{array}{l}\text { Mandaliya } \\
2019 \text { [24] }\end{array}$ & 2850 & $\begin{array}{l}1254 \\
(44 \%)\end{array}$ & $\begin{array}{l}\text { African } \\
\text { Ameri- } \\
\text { cans } \\
(57 \%), \\
\text { Cauca- } \\
\text { sians } \\
(13 \%)\end{array}$ & 61 & $\begin{array}{l}\text { Only good/ } \\
\text { excellent } \\
\text { prep }\end{array}$ & Excluded & NA & NA & $\begin{array}{l}\text { Surgeons: } \\
11.1 \text { min, } \\
\text { Academic Gl: } \\
9.1 \text { min, } \\
\text { Community } \\
\text { Gl: } 7.9 \text { min }\end{array}$ \\
\hline
\end{tabular}

States Multi-Society Task Force (USMSTF) has recognized the importance of these lesions and has recommended different polypectomy surveillance intervals based on the pathology and size of these polyps $[37,38]$. Our study attempted to derive pooled prevalence rates of SSL and proximal serrated polyps detected during screening colonoscopy that can be helpful for future studies to use as reference and examine if achieving this target also helps with reduction in post colonoscopy colon cancer. Recently another systematic review [39] examined clinical features and prevalence of SSL, however, the focus was not on the detection rates of these lesions during average risk screening colonoscopy.
Despite known contribution of SSL towards CIMP pathway of colonic malignancy, interval CRC cases and missed lesions [8, 40], SDR has not been investigated as a quality benchmark. Current guidelines are not able to suggest an SDR goal to achieve during screening colonoscopy due to two essential factors: first, lack of robust data providing real life prevalence of SDR in screening colonoscopy and second, lack of studies of higher SDR showing decrease in interval CRC. With surveillance guidelines in place, goals for SDR should be clarified for practicing endoscopists to track their performance. GI societies have unanimously recommended an ADR of $25 \%$ (men and women combined) for screening colonoscopy but a benchmark for SDR does not exist currently $[2,41]$. This could be due to several 


\section{Study name}

\begin{tabular}{lrrrr} 
& Event rate & Lower limit & Upper limit & Z-Value \\
\hline Chang 2016 & 0.014 & 0.012 & 0.018 & -39608 \\
Ross 2015 & 0.082 & 0.072 & 0.093 & -35274 \\
Sanaka 2014 & 0.018 & 0.013 & 0.025 & -24750 \\
Pereyra 2014 & 0.077 & 0.051 & 0.116 & -10921 \\
Hazewinkel 2014 & 0.048 & 0.038 & 0.060 & -24095 \\
Hetzel 2010 & 0.006 & 0.005 & 0.009 & -34112 \\
Buda 2012 & 0.023 & 0.016 & 0.035 & -17695 \\
Ladabaum 2016 & 0.012 & 0.005 & 0.026 & -10784 \\
ljspeert 2016 & 0.025 & 0.022 & 0.027 & -67011 \\
ljspeert (RCT) 2016 & 0.006 & 0.003 & 0.012 & -14283 \\
Schachschal 2016 & 0.007 & 0.003 & 0.014 & -13243 \\
Anderson 2018 & 0.033 & 0.029 & 0.038 & -44549 \\
Parikh 2017 & 0.043 & 0.037 & 0.050 & -40566 \\
Shaukat 2019 & 0.062 & 0.061 & 0.063 & -278064 \\
Schramm 2018 & 0.030 & 0.025 & 0.035 & -38203 \\
Mandaliya 2019 & 0.024 & 0.019 & 0.030 & -30238 \\
& 0.025 & 0.018 & 0.034 & -21502
\end{tabular}

Statistics for each study
P-Value

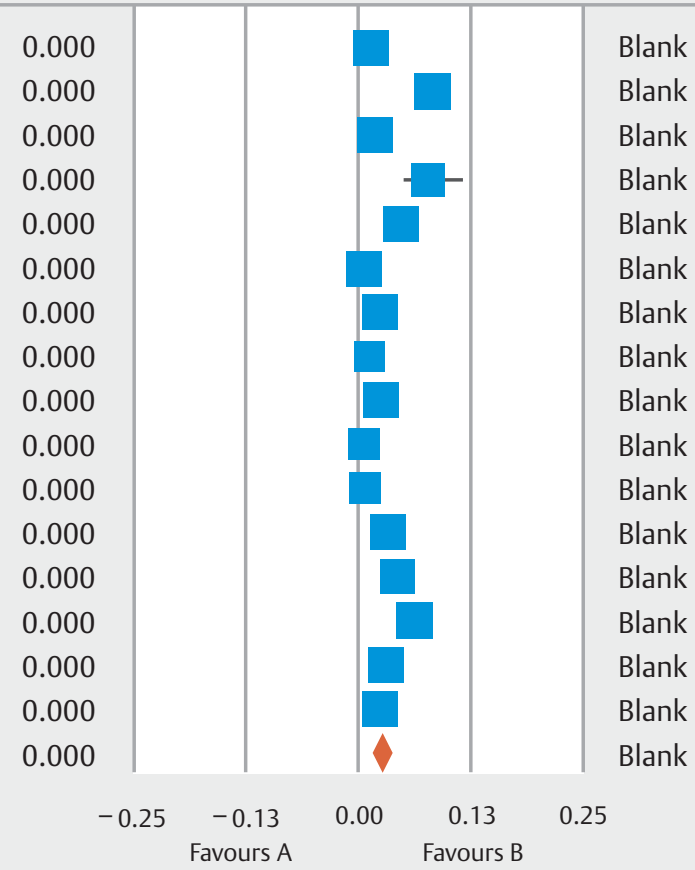

- Fig. 2 Forest plot of pooled rate of sessile serrated lesion detection rate (SDR).

\section{Study name}

Hazewinkel 2014

Leung 2012

Kahi 2011

Hetzel 2010

Buda 2012

ljspeert 2016

Schreiner 2010

Parikh 2017

Schramm 2018

Anderson 2017

de Wijkerslooth 2013

Mandaliya 2019

\section{. \\ Event rate Lower limit Upper limit}

$\begin{array}{llll}0.122 & 0.106 & 0.140 & -24392 \\ 0.072 & 0.059 & 0.087 & -23656 \\ 0.185 & 0.176 & 0.195 & -47029 \\ 0.095 & 0.089 & 0.102 & -56047 \\ 0.105 & 0.087 & 0.125 & -20623 \\ 0.097 & 0.092 & 0.102 & -77527 \\ 0.112 & 0.101 & 0.123 & -36495 \\ 0.061 & 0.054 & 0.069 & -42167 \\ 0.074 & 0.066 & 0.082 & -42667 \\ 0.108 & 0.105 & 0.112 & -113428 \\ 0.123 & 0.107 & 0.142 & -23730 \\ 0.090 & 0.080 & 0.101 & -35349 \\ 0.100 & 0.085 & 0.118 & -23046\end{array}$

\section{P-Value}

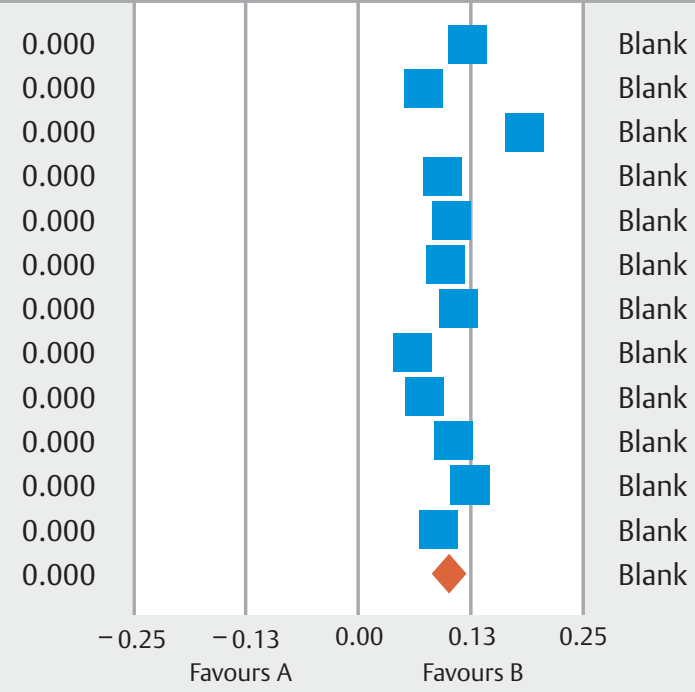

- Fig. 3 Forest plot of pooled rate of proximal serrated polyp detection rate (PSPDR).

factors including paucity of data on SSL, relatively recent awareness of the pre-malignant potential of these polyps in comparison to tubular adenomas, heterogeneity in the prevalence of SSL from various retrospective and prospective studies
[12], difficulty in identification of these polyps and endoscopic resection due to their inherent characteristics and poor interobserver agreement among pathologists in the diagnosis of SSL [42]. 
- Table 3 Pooled estimates of detection rates with heterogeneity of screening colonoscopy studies.

\begin{tabular}{|c|c|c|c|c|}
\hline Outcome & Reported by N studies & Pooled estimate (\%) ${ }^{*}$ & $95 \% \mathrm{Cl}$ & Heterogeneity (12) \\
\hline SDR & 16 & $2.5 \%$ & $1.8 \%-3.4 \%$ & $98.66 \%$ \\
\hline SDR for prospective studies & 7 & $2.3 \%$ & $1.5 \%-3.6 \%$ & $94.19 \%$ \\
\hline SDR for large studies $(n>1000)$ & 13 & $2.4 \%$ & $1.7 \%-3.4 \%$ & $98.89 \%$ \\
\hline SDR for prospective large studies & 4 & $2 \%$ & $1.1 \%-3.3 \%$ & $95.82 \%$ \\
\hline PSPDR & 12 & $10 \%$ & $8.5 \%-11.8 \%$ & $98.17 \%$ \\
\hline PSPDR for prospective studies & 4 & $11 \%$ & $9.6 \%-12.7 \%$ & $82.01 \%$ \\
\hline PSPDR for large studies $(n>1000)$ & 11 & $10 \%$ & $8.4 \%-11.9 \%$ & $98.34 \%$ \\
\hline PSPDR for prospective large studies & 3 & $11.2 \%$ & $9.3 \%-13.5 \%$ & $87.95 \%$ \\
\hline Any Serrated polyp detection rate & 10 & $22 \%$ & $14.9 \%-31.2 \%$ & $99.7 \%$ \\
\hline Large serrated polyp detection rate & 6 & $1.6 \%$ & $1.2 \%-2.2 \%$ & $89.87 \%$ \\
\hline Large SSL detection rate & 4 & $1.1 \%$ & $0.5 \%-2.2 \%$ & $95.53 \%$ \\
\hline Proximal SSL detection rate & 5 & $1.6 \%$ & $1.1 \%-2.4 \%$ & $93.59 \%$ \\
\hline SSL with dysplasia detection rate & 7 & $0.8 \%$ & $0.4 \%-1.6 \%$ & $92.78 \%$ \\
\hline TSA detection rate & 6 & $0.2 \%$ & $0.1 \%-0.6 \%$ & $82.64 \%$ \\
\hline Synchronous Advanced ADR & 16 & $19.1 \%$ & $15.6 \%-23.1 \%$ & $99.63 \%$ \\
\hline Clinically significant serrated polyp detection rate & 4 & $6.3 \%$ & $4.6 \%-8.5 \%$ & $98.4 \%$ \\
\hline
\end{tabular}

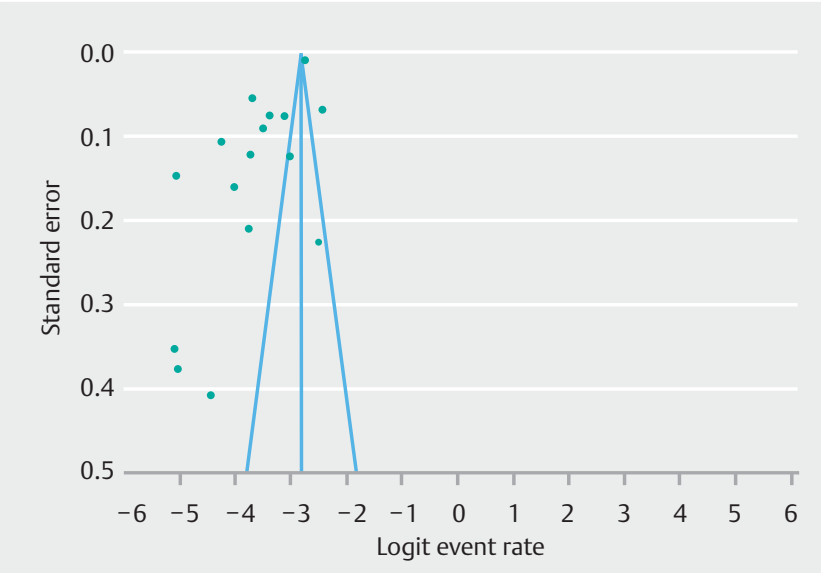

- Fig. 4 Funnel plot for SSL detection rate studies.

Several retrospective studies have shown a predilection of serrated polyps for the proximal colon $[43,44]$ and higher incidence of post colonoscopy colon cancers in the proximal colon has been attributed to possible missed serrated lesions. Rondagh et al. demonstrated that the serrated polyps in the proximal colon were more non-polypoid in comparison to distal polyps making them easier to be missed [45]. In addition, the pathological diagnosis of SSL has a significant interobserver variability among pathologists. Gourewitch et al [42] showed that there was significant variation in the pathological interpre- tation of SSL compared to adenomas. Comparing pathologists with lowest versus highest classification rates for SSL and adenomas, the SSL prevalence varied from $0.5 \%$ to $12 \%$ compared to $28.5 \%$ to $42.4 \%$ for adenomas. This has also made it difficult to estimate the true prevalence rate of SSL. In addition, change in classification and identification of SSL (previously SSP/A) has also contributed towards precise diagnosis of SSLs over time.

Recently, SDR has been correlated with ADR by few studies and that ADR could be used as a surrogate marker [12, 26, 46]. However, there has been a concern expressed by some experts that endoscopists who meet the ADR benchmark may not necessarily have an adequate SDR. In a retrospective cohort of more than 2000 patients, Sanaka et al [27] reported an SDR of $2 \%$ with proximal SDR three times higher than the distal colon ( $1.4 \%$ vs $0.5 \%$ ) but found a poor correlation between SDR and ADR (correlation co-efficient, $r=0.35$ ). Another potential important benchmark is the clinically significant serrated polyp detection rate [5] since reporting SDR alone would not assist prescribing surveillance interval when only proximal $H P>5 \mathrm{~mm}$ is found. A recent study [47] examined correlation of ADR and clinically significant serrated polyp detection rate among endoscopists with ADR greater than $25 \%$. There was a significant positive but modest correlation between these two parameters. These studies question the reliability of ADR as an actual surrogate marker for SDR or clinically significant serrated polyp detection rate and further emphasize the need for an actual benchmark for SDR. Rather than using surrogate markers, pooled rates from the current systematic review and meta-a- 
nalysis could be used to determine if endoscopists reaching an ADR of $30 \%$ or higher are as good at the detection of serrated lesions.

Strengths of this current analysis include the use of robust data from two RCTs $[19,21]$ and six prospective studies [17, $18,22,23,31,36]$ from different countries and restricting data collection to individuals undergoing average risk screening colonoscopy only. However, there are certain limitations. There was very high heterogeneity found in all outcomes from the pooled analysis. We attempted to examine only larger studies $(n \geq 1000)$ and prospective studies in sensitivity analysis in attempt to explore it but still there was considerable heterogeneity. This could be from varying sample sizes, various definitions used over years, use of adjunct modalities for electronic chromoendoscopy and/or distal attachments and most being single arm studies. Unfortunately, exploration of clinical and methodological heterogeneity cannot be performed due to lack of data on them for this analysis. While this is certainly reflect heterogenous data but real-world prevalence of any parameter would also follow a heterogenous distribution when assessed in populations spread over several continents raising questions for future studies to be answered. Finding publication bias in the available data also supports high heterogeneity and likely from different factors including studies of varying sizes and variation in detection and reporting over years. The wide range of prediction interval we noted also correlates with high heterogeneity in the available data.

In addition, only a few studies clearly report the distinction of various serrated lesions into SSL and others; this may have led to under representation of the actual data (true prevalence). Another inherent and well-established issue is the interobserver variation among pathologist in interpretation of SSL. This will be difficult to control for in any future multicenter studies as well unless there is central pathology processing of data with structured teaching. Only a few studies reported cecal intubation rates and bowel preparation information, both of which are intrimately linked with ADR and potentially with SDR. Also, several studies were conducted by expert and experienced endoscopists with high ADRs. These factors should be accounted for when results are interpreted. While gender is not an important predictor of SDR [32], we did not have information from studies to report separate SDR based on gender. Definitions of serrated lesions have been changing over the last several years and that may cause rates to differ a bit in any future robust trial. However, we used only pathology-confirmed data from inclusion studies when possible. Differences in colonoscopy withdrawal patterns (time required or taken, and techniques used, i.e. retroflexion in the right colon) and its impact on SDR is important. We attempted to include this pertinent information from inclusion studies. However, this was not the primary focus of these studies and so its impact on pooled SDR and PSPDR could not be determined. Also, we were not able to obtain adequate information from these studies done over time to examine if concurrent use of electronic chromoendoscopy modalities like narrow band imaging (NBI) and others could have contributed to higher detection of serrated polyps over years.

\section{Conclusion}

In conclusion, the current study provides pooled estimates of SSL and proximal serrated polyp detection rates for average risk screening colonoscopy from the available literature. These data may provide a reference for future prospective studies with clear SSL definitions to confirm precise SSL rates for screening colonoscopy. This has implications for assessing practicing endoscopists and trainees in terms of the quality of colonoscopy they can perform. We anticipate future research geared toward establishing robust evidence to identify precise SDR rates that are valid, generalizable, and reliable and that can be shown to reduce interval cancer rates, which will improve the quality of screening colonoscopy.

\section{Competing interests}

The authors declare that they have no conflict of interest.

\section{References}

[1] Siegel RL, Miller KD, Jemal A. Cancer statistics, 2015. CA Cancer J Clin 2015; 65: 5-29

[2] Rex DK, Petrini JL, Baron TH et al. Quality indicators for colonoscopy. Am J Gastroenterol 2006; 101: 873-885

[3] Kaminski MF, Wieszczy P, Rupinski M et al. Increased rate of adenoma detection associates with reduced risk of colorectal cancer and death. Gastroenterology 2017; 153: 98-105

[4] Hassan C, Repici A. Defeating cancer by boosting the adenoma detection rate: the circle of life. Gastroenterology 2017; 153: 8-10

[5] Rex DK, Ahnen DJ, Baron JA et al. Serrated lesions of the colorectum: review and recommendations from an expert panel. Am J Gastroenterol 2012; 107: 1315-1329; quiz 1314, 1330

[6] Patai AV, Molnar B, Tulassay Z et al. Serrated pathway: alternative route to colorectal cancer. World J Gastroenterol 2013; 19: 607-615

[7] Hawkins N], Bariol C, Ward RL. The serrated neoplasia pathway. Pathology 2002; 34: 548-555

[8] Benedict M, Galvao Neto A, Zhang X. Interval colorectal carcinoma: An unsolved debate. World J Gastroenterol 2015; 21: 12735-12741

[9] Nishihara R, Wu K, Lochhead P et al. Long-term colorectal-cancer incidence and mortality after lower endoscopy. N Engl J Med 2013; 369: 1095-1105

[10] JE IJ, Vermeulen L, Meijer GA et al. Serrated neoplasia-role in colorectal carcinogenesis and clinical implications. Nat Rev Gastroenterol Hepatol 2015; 12: 401-409

[11] Yamane L, Scapulatempo-Neto C, Reis RM et al. Serrated pathway in colorectal carcinogenesis. World J Gastroenterol 2014; 20: 26342640

[12] Ohki D, Tsuji Y, Shinozaki T et al. Sessile serrated adenoma detection rate is correlated with adenoma detection rate. World J Gastrointest Oncol 2018; 10: 82-90

[13] Moher D, Shamseer L, Clarke M et al. Preferred reporting items for systematic review and meta-analysis protocols (PRISMA-P) 2015 statement. Syst Rev 2015; 4: 1

[14] Wells G, Shea B, O'Connell D et al. The Newcastle-Ottawa Scale (NOS) for assessing the quality of nonrandomised studies in meta-analyses. 2013 
[15] Higgins JP, Altman DG, Gotzsche PC et al. The Cochrane Collaboration's tool for assessing risk of bias in randomised trials. BMJ 2011; 343: d5928

[16] Anderson JC, Butterly LF, Robinson CM et al. Risk of metachronous high-risk adenomas and large serrated polyps in individuals with serrated polyps on index colonoscopy: data from the New Hampshire Colonoscopy Registry. Gastroenterology 2018; 154: 117-127 e112

[17] Buda A, De Bona M, Dotti I et al. Prevalence of different subtypes of serrated polyps and risk of synchronous advanced colorectal neoplasia in average-risk population undergoing first-time colonoscopy. Clin Transl Gastroenterol 2012; 3: e6

[18] Chang LC, Shun CT, Hsu WF et al. Fecal Immunochemical test detects sessile serrated adenomas and polyps with a low level of sensitivity. Clin Gastroenterol Hepatol 2017; 15: 872-879 e871

[19] Hazewinkel Y, de Wijkerslooth TR, Stoop EM et al. Prevalence of serrated polyps and association with synchronous advanced neoplasia in screening colonoscopy. Endoscopy 2014; 46: 219-224

[20] Hetzel JT, Huang CS, Coukos JA et al. Variation in the detection of serrated polyps in an average risk colorectal cancer screening cohort. Am J Gastroenterol 2010; 105: 2656-2664

[21] JE I], Tutein Nolthenius C], Kuipers EJ et al. CT-colonography vs colonoscopy for detection of high-risk sessile serrated polyps. Am J Gastroenterol 2016; 111: 516-522

[22] JEG I], Bevan R, Senore C et al. Detection rate of serrated polyps and serrated polyposis syndrome in colorectal cancer screening cohorts: a European overview. Gut 2017; 66: 1225-1232

[23] Ladabaum U, Patel A, Mannalithara A et al. Predicting advanced neoplasia at colonoscopy in a diverse population with the National Cancer Institute colorectal cancer risk-assessment tool. Cancer 2016; 122: 2663-2670

[24] Mandaliya R, Baig K, Barnhill M et al. Significant Variation in the detection rates of proximal serrated polyps among academic gastroenterologists, community gastroenterologists, and colorectal surgeons in a single tertiary care center. Dig Dis Sci 2019; 64: 2614-2621

[25] Parikh MP, Muthukuru S, Jobanputra Y et al. Proximal Sessile serrated adenomas are more prevalent in Caucasians, and gastroenterologists are better than nongastroenterologists at their detection. Gastroenterol Res Pract 2017: doi:10.1155/2017/6710931

[26] Ross WA, Thirumurthi S, Lynch PM et al. Detection rates of premalignant polyps during screening colonoscopy: time to revise quality standards? Gastrointest Endosc 2015; 81: 567-574

[27] Sanaka MR, Gohel T, Podugu A et al. Adenoma and sessile serrated polyp detection rates: variation by patient sex and colonic segment but not specialty of the endoscopist. Dis Colon Rectum 2014; 57 : 1113-1119

[28] Schachschal G, Sehner S, Choschzick M et al. Impact of reassessment of colonic hyperplastic polyps by expert GI pathologists. Int J Colorectal Dis 2016; 31: 675-683

[29] Schramm C, Janhsen K, Hofer JH et al. Detection of clinically relevant serrated polyps during screening colonoscopy: results from seven cooperating centers within the German colorectal screening program. Endoscopy 2018; 50: 993-1000

[30] Shaukat A, Gravely AA, Kim AS et al. Rates of detection of adenoma, sessile serrated adenoma, and advanced adenoma are stable over time and modifiable. Gastroenterology 2019; 156: 816-817

[31] de Wijkerslooth TR, Stoop EM, Bossuyt PM et al. Differences in proximal serrated polyp detection among endoscopists are associated with variability in withdrawal time. Gastrointest Endosc 2013; 77: 617-623
[32] Kahi C], Hewett DG, Norton DL et al. Prevalence and variable detection of proximal colon serrated polyps during screening colonoscopy. Clin Gastroenterol Hepatol 2011; 9: 42-46

[33] Leung WK, Tang V, Lui PC. Detection rates of proximal or large serrated polyps in Chinese patients undergoing screening colonoscopy. J Dig Dis 2012; 13: 466-471

[34] Schreiner MA, Weiss DG, Lieberman DA. Proximal and large hyperplastic and nondysplastic serrated polyps detected by colonoscopy are associated with neoplasia. Gastroenterology 2010; 139: 14971502

[35] Anderson JC, Butterly LF, Weiss JE et al. Providing data for serrated polyp detection rate benchmarks: an analysis of the New Hampshire Colonoscopy Registry. Gastrointest Endosc 2017; 85: 1188-1194

[36] Pereyra L, Gomez EJ, Gonzalez R et al. Finding sessile serrated adenomas: is it possible to identify them during conventional colonoscopy? Dig Dis Sci 2014; 59: 3021-3026

[37] Lieberman DA, Rex DK, Winawer S] et al. Guidelines for colonoscopy surveillance after screening and polypectomy: a consensus update by the US Multi-Society Task Force on Colorectal Cancer. Gastroenterology 2012; 143: 844-857

[38] Gupta S, Lieberman D, Anderson JC et al. Recommendations for Follow-up after colonoscopy and polypectomy: a consensus update by the US Multi-Society Task Force on Colorectal Cancer. Gastrointest Endosc 2020; 91: 463-485 e465

[39] Meester RGS, van Herk M, Lansdorp-Vogelaar I et al. Prevalence and clinical features of sessile serrated polyps: a systematic review. Gastroenterology 2020; 159: 105-118 e125

[40] Burgess NG, Tutticci NJ, Pellise M et al. Sessile serrated adenomas/ polyps with cytologic dysplasia: a triple threat for interval cancer. Gastrointest Endosc 2014; 80: 307-310

[41] Kaminski MF, Thomas-Gibson S, Bugajski M et al. Performance measures for lower gastrointestinal endoscopy: a European Society of Gastrointestinal Endoscopy (ESGE) quality improvement initiative. United Europ Gastroenterol ] 2017; 5: 309-334

[42] Gourevitch RA, Rose S, Crockett SD et al. Variation in pathologist classification of colorectal adenomas and serrated polyps. Am J Gastroenterol 2018; 113: 431-439

[43] Gurudu SR, Heigh RI, De Petris G et al. Sessile serrated adenomas: demographic, endoscopic and pathological characteristics. World J Gastroenterol 2010; 16: 3402-3405

[44] Spring KJ, Zhao ZZ, Karamatic R et al. High prevalence of sessile serrated adenomas with BRAF mutations: a prospective study of patients undergoing colonoscopy. Gastroenterology 2006; 131: 1400-1407

[45] Rondagh EJ, Bouwens MW, RiedI RG et al. Endoscopic appearance of proximal colorectal neoplasms and potential implications for colonoscopy in cancer prevention. Gastrointest Endosc 2012; 75: 12181225

[46] Zorzi M, Senore C, Da Re F et al. Detection rate and predictive factors of sessile serrated polyps in an organised colorectal cancer screening programme with immunochemical faecal occult blood test: the EQuIPE study (Evaluating Quality Indicators of the Performance of Endoscopy). Gut 2017; 66: 1233-1240

[47] Klair JS, Ashat M, Johnson D et al. Serrated polyp detection rate and advanced adenoma detection rate from a US multicenter cohort. Endoscopy 2019: doi:10.1055/a-1031-5672 